\title{
Molecular and hydrodynamic properties of human epidermal growth factor receptor HER2 extracellular domain and its homodimer: Experiments and multi-scale simulations
}

\author{
J.F. Vega ${ }^{1 *}$, J. Ramos ${ }^{1}$, V. Cruz ${ }^{l}$, E. Vicente-Alique ${ }^{I}$, E. Sánchez-Sánchez ${ }^{l}$, A. Sanchez-Fernández ${ }^{l}, Y$. \\ Wang, ${ }^{4}$ P. Hu, ${ }^{4}$ J. Cortés ${ }^{2,3}$ and J. Martínez-Salazar ${ }^{1}$
}

${ }^{1}$ BIOPHYM, Department of Macromolecular Physics, Instituto de Estructura de la Materia, IEMCSIC, C/ Serrano 113 bis, 28006 Madrid, Spain

${ }^{2}$ Ramon y Cajal University Hospital, Ctra. de Colmenar Viejo, km 9,100, 28034 Madrid, Spain

${ }^{3}$ Vall D'Hebron Institute of Oncology (VHIO), Paseo Vall Hebron 119-129, 08035 Barcelona, Spain

${ }^{4}$ Sino Biological, Inc., Beijing, People's Republic of China

*e-mail: jf.vega@csic.es

\begin{abstract}
Background: In a broad range of human carcinomas gene amplification leads to HER2 overexpression, which has been proposed to cause spontaneous dimerization and activation in the absence of ligand. This makes HER2 attractive as a therapeutic target. However, the HER2 homodimerization mechanism remains unexplored. It has been suggested that the "back-to-back" homodimer does not form in solution. Notwithstanding, very recently the crystal structure of the HER2 extracellular domain homodimer formed with a "back-to-head" interaction has been resolved. We intend to explore the existence of such interactions.

Methods: A combination of experiments, molecular dynamics and hydrodynamic modeling were used to monitor the transport properties of HER2 in solution.

Results \& conclusions: We have detected the HER2 extracellular domain homodimer in solution. The results show a high degree of molecular flexibility, which ultimately leads to quite higher values of the intrinsic viscosity and lower values of diffusion coefficient than those corresponding to globular proteins. This flexibility obeys to the open conformation of the receptor and to the large fluctuations of the different domains. We also report that for obtaining the correct hydrodynamic constants from the modeling one must consider the glycosylation of the systems.

General significance: Conformational features of epidermal growth factor receptors regulate their hydrodynamic properties and control their activity. It is essential to understand the dynamics of these systems and the role of the specific domains involved. To find biophysical correlations between dynamics and macroscopic transport properties is of general interest for researches working in this area.
\end{abstract}

Keywords: HER2 homodimer, hydrodynamic properties, molecular dynamics, hydrodynamic modeling, conformational fluctuations. 


\section{Introduction}

There are four members in the human epidermal growth factor receptor (EGFR) family: ErbB1/EGFR (HER1), ErbB2 (HER2), ErbB3 (HER3) and ErbB4 (HER4). The structure of these receptors consists of three differentiated regions: an extracellular ligandbinding domain (ECD) containing four sub-domains (I-IV), a single transmembrane $\alpha$ - helix (TMH) spanning both the cellular membrane and the intracellular juxtamembrane (IJM), and an intracellular tyrosine-kinase (TK) domain. The ECD of HER1, HER3 and HER4 contains a specific region that binds ligands. It is recognized that the ligand-dependent homo and heterodimerization of ECD and the consequent induction of the tyrosine-kinase activity of its intracellular domain is the key factor controlling HERs signaling, which in turns regulates multiple cellular processes including cellular growth and proliferation, survival, differentiation and migration [1]. The dimerization occurs via a conformational change from a tethered to an extended-exposed arm structure, which facilitates the contact between the ECD of two monomers [2-4]. However, unlike the other members of the family, HER2 does not bind any ligand andis able to adopt a fixed extended conformation, resembling the ligandactivated state, being able to [5, 6], form HER2-HER1/3/4 heterodimers [7].

In a broad range of human carcinomas, gene amplification leads to HER2 overexpression, which has been proposed to cause spontaneous receptor dimerization [8]. This has made HER2 especially attractive as a therapeutic target [9]. In this context, however, the mechanism triggering molecular HER2 homodimerization still remains under discussion. It has been repeatedly reported that the "back-to-back" HER2 homodimer is either very weak or does not form at all in solution [10-13]. Notwithstanding, the crystal structure of the HER2 ECD homodimer as a "back-to-head" configuration of interaction has been recently resolved [14]. The results suggest that HER2 dimerization is different from the homodimerization model for HER1 ECD. The elucidation of this interaction is important, not only for understanding the mechanism of the very elusive, ligand-independent HER2 homodimerization but also for getting insight of potential therapeutic function at molecular level.

To the best of our knowledge, the conformational features and molecular dynamics of HER2 ECD domain in solution have not been investigated in detail. This report attempts to use a combination of experimental hydrodynamic measurements, molecular dynamics (MD) simulation and continuum hydrodynamics modeling to explore HER2 ECD behavior in aqueous solutions. The measured HER2 ECD molecular mass indicates that the receptor 
ectopic domain mainly exists as monomer in solution under the experimental conditions used. Nevertheless, the results suggest the existence of a measurable amount of homodimer in solution (5 - $15 \%$ of the total amount by weight). In addition, enhanced values of intrinsic viscosities with respect to those found in typical proteins are reported. The open configuration of the receptor and the strong conformational fluctuations, mainly due to domain IV, might be the cause of these enhanced values. We have also found that the inclusion of the carbohydrate chains in the computational model is critical in order to achieve good correlation between experimental and simulated properties. The combined use of experiments and simulations reveal the true dynamics of the systems in solution, an insight beyond what is known from the crystal of partial structures.

\section{Materials and methods}

\subsection{Production and purification of HER2-his, HER2 and Trastuzumab}

The HER2-his tag and HER2 ECD samples were provided by Sino Biological, Inc. (Beijing, China). For proteins construction a DNA sequence encoding the extracellular domain (Met 1-Thr 652) of human HER2 (NP_004439.2) was fused with the polyhistidine tag (g-eHER2-his) or with five amino acids, DDDDK (g-eHER2), at the C-terminus. The proteins were produced in human cells. The recombinant human g-eHER2-his comprises 641 amino acids and has a calculated molecular mass of $70.7 \mathrm{kDa}$. The recombinant human g-eHER2 consists of 636 amino acids with a predicted molecular mass of $70.0 \mathrm{kDa}$. As a result of glycosylation, proteins migrate to a value of approximately 100-110 kDa in SDS-PAGE under reducing conditions. After multi-step purification process based on chromatography and filtration, the purified products can reach at least $95 \%$ purity, and stored as lyophilized powder from sterile PBS ( $\mathrm{pH}$ 7.4). $5 \%-8 \%$ trehalose and mannitol are added as protectants before lyophilization. We have also studied as a model monodisperse system, the trastuzumab (TZM) Herceptin ${ }^{\odot}$ (stock solution at $21 \mathrm{mg} \cdot \mathrm{mL}^{-1}$ ) that was kindly provided by one of us ( $\mathrm{J}$. Cortés).

\subsection{Preparation of protein solutions}

Desalting and buffer exchange have been carried out using centrifugal concentrators (Amicon Ultra-0.5 mL, Merck Millipore, Billerica, USA). Both HER2 ECD proteins and TZM antibody were finally filtered (Millex-GV $0.22 \mu \mathrm{m}$, Merck Millipore, Billerica, USA) and stored in $20 \mathrm{mM}$ Tris- $\mathrm{HCl} \mathrm{pH} 7.5,150 \mathrm{mM} \mathrm{NaCl}$ for subsequent analysis of their 
hydrodynamic properties. Water for all buffers and dilutions was obtained from Milli-Q water purification system (Merck Millipore, Billerica, USA). As control systems we have used a set of globular proteins (BSA and aldolase, GE Healthcare, Buckinghamshire, UK).

\subsection{Size Exclusion Chromatography and Tetradetection: Protein concentration, molecular mass and intrinsic viscosity}

The determination of the concentration, absolute molecular weight and hydrodynamic properties of the proteins in solution was carried out by size exclusion chromatography (SEC) coupled with tetradetection using a GPCmax-TDA system (Viscotek and Malvern Instruments, Worcestershire, UK) at $\mathrm{T}=293 \mathrm{~K}$. The GE Superdex ${ }^{\mathrm{TM}} 200$ column (GE Healthcare, Buckinghamshire, UK) for SEC was equilibrated with a buffer composed of 20 $\mathrm{mM}$ Tris $\mathrm{pH} 7.5,150 \mathrm{mM} \mathrm{NaCl} .50$ to $100 \mu \mathrm{L}$ of sample at a concentration of $1-3 \mathrm{mg} \cdot \mathrm{mL}^{-1}$ were injected into the SEC column and eluted with the equilibration buffer at a flow rate of $0.25 \mathrm{~mL} \cdot \mathrm{min}^{-1}$. Elution profiles were followed by a UV-photo diode array (UV-PDA), a differential refractometer (RI), a $7^{\circ}$ low angle light scattering detector (LALS), a $90^{\circ}$ right angle light scattering detector (RALS) and a differential pressure viscometer (DP). Bovine serum albumin (BSA) was used as a standard reference protein of known molecular weight, concentration, refractive index increment $\left(\mathrm{dn} / \mathrm{dc}=0.185 \mathrm{~mL} \cdot \mathrm{g}^{-1}\right)$, and intrinsic viscosity. In such a way, the instrument response factors of the detectors for the mobile phase were determined. The OmniSEC software program was used for the acquisition and analysis of the data. From the experimental absolute molecular weight and intrinsic viscosity data, the hydrodynamic size of the systems was determined. The hydrodynamic radius $\mathrm{r}_{\mathrm{h}}$ was calculated according to the Einstein viscosity relation, modelling the hydrated polymer molecules in terms of equivalent hydrodynamic spheres that would increase the viscosity to the same extent as solid spherical particles of volume $V_{\mathrm{e}}$ :

$$
[\eta]=\frac{2.5 N_{A} V_{e}}{M_{w}}
$$

where $M_{w}$ is the polymer molecular weight, $N_{A}$ is the Avogadro's number, and $V_{e}$ is the volume of the equivalent spherical particle. Since $V_{e}=4 / 3 \pi r_{h}{ }^{3}$; rearrangement of Eq. 1 gives:

$$
r_{h}=\left(\frac{3[\eta] M_{w}}{10 \pi N_{A}}\right)^{1 / 3}
$$




\subsection{Dynamic light scattering: Diffusion coefficient}

Dynamic light scattering (DLS) electric field correlations have been obtained using the Zetasizer Nano ZS (Malvern Instruments, Worcestershire, UK) at T = $293 \mathrm{~K}$, equipped with a $12 \mu \mathrm{L}$ quartz batch cuvette (Malvern Instruments ZEN2112). The concentration of the samples was adjusted to $\mathrm{c}$ values $<1 \mathrm{mg} \cdot \mathrm{mL}^{-1}$, to avoid the effect of particle-particle interactions on the diffusion coefficient. Internal control of the experiments was performed with aqueous solutions of standard polystyrene beads (Thermo Scientific Inc., Waltham, MA, USA and Malvern Instruments, Worcestershire, UK). The Nano ZS instrument incorporates noninvasive backscattering (NIBS) optics and homodyne detection, to avoid artifacts in the determination of the particles size. This technique measures the time-dependent fluctuations in the intensity of scattered light that occur because particles in solution undergo Brownian motion. The intensity fluctuations measured are used to produce the scattered intensity time correlation function, $g_{2}(\tau)$. The time-dependence autocorrelation function of the photocurrent was acquired in the solutions, every $10 \mathrm{~s}$, with 15 acquisitions for each run. The sample solution was illuminated with a $\lambda_{0}=633 \mathrm{~nm}$ laser at constant power, and the intensity of light scattered at an angle of $\theta=173^{\circ}$ was measured by an avalanche photodiode. The Siegert equation expresses the relation between the normalized time correlation function of the scattered intensity $g_{2}(\tau)$ and the normalized time correlation function of the electric field $g_{1}(\tau)$ [15]:

$$
g_{2}(\tau)=B+\beta\left[g_{1}(\tau)\right]^{2}
$$

where $\tau$ is the lag time, $\mathrm{B}$ is the baseline and $\beta(\leq 1)$ is a coherence factor that accounts for deviations from ideal correlation and the experimental geometry.

In the ideal case of a monodisperse species it is possible to treat the first order autocorrelation function as a single exponential decay:

$$
g_{1}(\tau)=e^{(-\tau)}
$$

where $\Gamma=\mathrm{D} \cdot \mathrm{q}^{2}$ is the average decay rate with $\mathrm{D}$ being the diffusion coefficient and $\mathrm{q}=$ $\left(4 \pi \mathrm{n} / \lambda_{0}\right) \sin (\theta / 2)$ the corresponding magnitude of the scattering vector, $\mathrm{n}$ the refractive index 
of solution, $\lambda_{0}$ the wavelength of the laser, and $\theta$ the scattering angle. The cumulant analysis allows one to determine the first few moments of the distribution. The corresponding cumulant for the field autocorrelation function can be written as:

where $\Gamma=D_{z} \cdot q^{2}$ is the average decay rate with $D_{z}$ being the z-average diffusion coefficient. In Eq. 5, $\mu_{2}$ is the variance of the distribution, and the polydispersity index is defined as $\mathrm{Q}=$ $\mu_{2} \cdot \Gamma^{-2}$.

A more complicated regularization fitting scheme may be used to find broad distributions of particle sizes. In our case, the autocorrelation function of the scattered intensity was analyzed by means of the inverse Laplace transform by the Thikonov analysis using the program SEDFIT (http://www.AnalyticalUltracentrifugation.com) for obtaining the decay rate distribution from the intensity [16]. Once the decay rate $\Gamma$ distribution is obtained, it is used to obtain the size distribution by taking advantage of the Stokes-Einstein relationship that relates $\Gamma$ and, alternatively, the diffusion coefficient, $\mathrm{D}$, to the hydrodynamic radius, $\mathrm{r}_{\mathrm{h}}$, of the object that undergoes translational motion as:

$$
r_{h}=\frac{k_{B} T}{6 \pi \eta D}
$$

In Eq (6), $\mathrm{k}_{\mathrm{B}}$ is the Boltzmann's constant, $\mathrm{T}$ is the absolute temperature ( $\left.\mathrm{T}=293 \mathrm{~K}\right)$, and $\eta$ is the solvent viscosity, $\eta=1.023 \mathrm{mPa} \cdot \mathrm{s}$. This equation considers the particles as rigid spheres with a diameter related to the translational diffusion coefficient, which in this instance depends on the size and conformation of the particle at a given temperature and solvent viscosity. All this process allows one to obtain the size distribution by analysis of the intensity. The size distribution by weight, obtained from the application of Mie theory, was used for the interpretation of the results [17]. The results obtained with SEDFIT compare very well with the obtained from commercial software [18]. It should be note here that the values of $r_{h}$ given by Eqs (2) and (6) are equivalent only in the case of spherical rigid particles [19]. In the simplest case for most of proteins one can define the ellipsoid of revolution to describe their shapes. Calculations allow obtaining the equivalent radius from different hydrodynamic properties as a function of $\mathrm{p}=\mathrm{L} / \mathrm{d}$, the aspect ratio of the ellipsoids, being $L$ and $d$ the largest and the smallest dimension, respectively [19]. The values obtained 
for $r_{h}$ from $[\eta]$ and $D$ in this simple case are nearly the same for a wide range of $p$ values, from flat disks $(\mathrm{p}=0.1)$ to long rods $(\mathrm{p}=10)$.

\subsection{Computational simulations}

\subsubsection{Construction of the models}

The simulated systems are listed in Table 1 and the initial coordinates are given as PDB files in the Supplementary Information section.

Table 1. Systems studied by computer simulations

\begin{tabular}{|c|c|c|c|c|}
\hline System & $\mathbf{N}_{\text {traj }}$ & $\begin{array}{l}\mathbf{t}_{\mathrm{MD}} \\
\text { (ns) }\end{array}$ & $\begin{array}{c}\mathbf{M}_{\mathbf{w}} \\
(\mathbf{k D a})\end{array}$ & Description \\
\hline TZM & 8 & 50 & 149.0 & $\begin{array}{l}\text { Trastuzumab antibody model including } \\
\text { oligosaccharide residues published by Brandt } \\
\text { et al. [20] }\end{array}$ \\
\hline eHER2-his & 8 & 50 & 70.7 & HER2 ECD domain with histidine tag \\
\hline g-eHER2-his & 8 & 50 & 85.6 & $\begin{array}{l}\text { Glycosylated HER2 ECD domain with } \\
\text { histidine tag }\end{array}$ \\
\hline g-eHER2-his dimer & 8 & 50 & 171.2 & $\begin{array}{l}\text { Crystallographic dimer }[14] \text { adding the } \\
\text { glycosylation pattern used in the monomer. }\end{array}$ \\
\hline
\end{tabular}

The Trastuzumab model (TZM). The initial coordinates for TZM are directly obtained from Brandt et al. [20]. Fig. 1a shows a representation of the simulated structure. The calculated antibody molecular weight $(148.9 \mathrm{kDa})$ is in line with the experimentally determined one. 

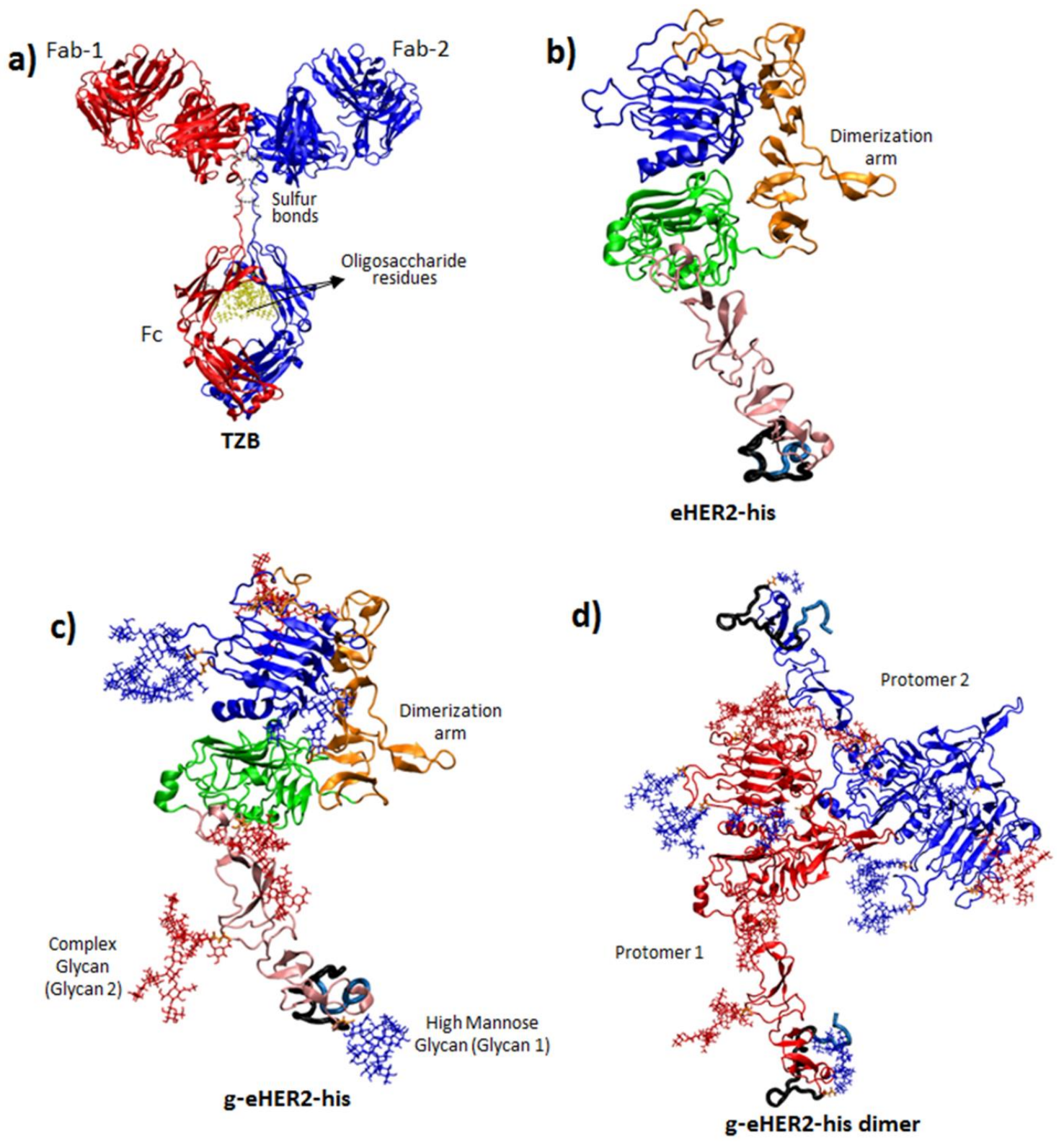

Fig. 1. Initial structures of the proteins studied in this work. a) Trastuzumab: Chains are colored in red (residues 1-664) and blue (residues 665-1328), respectively. The glycan chains and sulfur bonds are shown in yellow and gray CPK, respectively. b) Extracellular domain of the HER2 protein tagged with histidine: The domain I (residues 23-195), II (residues 196-339), III (residues 340-510) and IV (residues 511-629) are in blue, orange, green and pink, respectively. The domain IV loop 630-652 and the histidine tag (653-662) are highlighted in black and blue, respectively (see text for details). c) Glycosylated g-eHER2-his: The residues $\mathbf{N}^{\mathbf{6 8}}, \mathbf{N}^{\mathbf{1 2 4}}, \mathbf{N}^{\mathbf{2 5 9}}$ and $\mathbf{N}^{\mathbf{6 2 9}}$ are $\mathrm{N}$-glycosylated with the glycan 1 (blue sticks) and $\mathbf{N}^{\mathbf{1 8 7}}, \mathbf{N}^{\mathbf{5 3 0}}$ and $\mathbf{N}^{\mathbf{5 7 1}}$ with the glycan 2(red sticks). d) Glycosylated g-eHER2-his dimer: The monomers are colored in red and blue

The human HER2 extracellular domain with histidine tag model (eHER2-his). This model comprises 640 amino acids with a molecular mass of $70.7 \mathrm{kDa}$. The initial structure of the HER2 protein ECD (residues 23-629) was obtained from the Protein Data Bank Server (PDB ID: 1NZ8) and prepared as explained in our previous work [21, 22]. The loop defined by the residues 630 to 652 (in bold in Fig. 1b) was missing in the crystallographic structure 
and then it was modeled using the loop refinement algorithms implemented in the Prime application from the Schrodinger's Maestro package [23]. After this, the histidine tag (residues 653-662) was built using a similar approach. Each loop was independently refined using the serial loop sampling with the ultra-extended sampling method with an energy cutoff of $10.0 \mathrm{kcal} \cdot \mathrm{mol}^{-1}$. An extra refinement on the side-chain of the residues within $7.5 \AA$ around the loop was applied to eliminate bad contacts. Finally, a truncated-Newton energy minimization on these loops was achieved using the OPLS_2005 force field, keeping constrained the rest of the protein. The initial structure used in this work is shown in Fig. $1 \mathrm{~b}$.
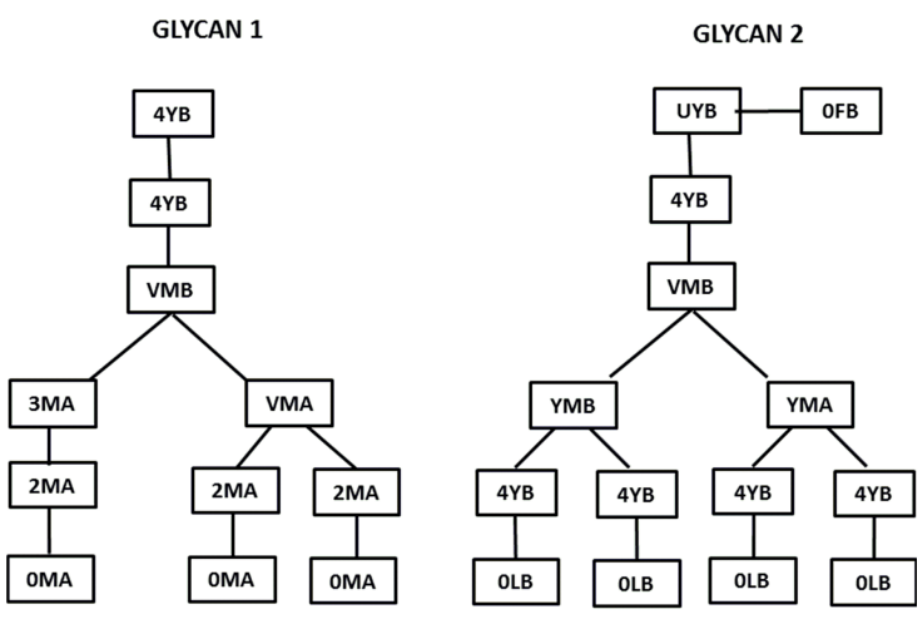

\begin{tabular}{|cc|}
\hline Amber Code & Saccharide Name \\
\hline OLB & D-Galactose $\Delta$ \\
\hline UYB & D-N-acetylglucosamine \\
\hline 4YB & D-N-acetylglucosamine \\
\hline VMB & $3,6-\beta$-Manose \\
\hline YMB & $2,4-\beta$-Manose \\
\hline OMA & $\alpha$-Manose \\
\hline 3MA & $\alpha$-3-Manose \\
\hline 2 MA & $\alpha-2$-Manose \\
\hline VMA & $3,6-\alpha$-Manose \\
\hline YMA & $1,6-\alpha$-Manose \\
\hline OFB & Fucose \\
\hline
\end{tabular}

Scheme 1. Oligosaccharide composition used in the g-eHER2-his model. Upper panel: Bonding scheme for the two glycan structures used in the model. Lower panel: GLYCAM06 AMBER saccharide codes along with their chemical names

The glycosylated human HER2 extracellular domain with histidine tag (g-eHER2-his) model. The eHER2-his protein is known to be glycosylated (g-eHER2-his) with a measured molecular weight of $88.7 \pm 1.8 \mathrm{kDa}$. The theoretical molecular weight from the amino acid sequence is $70.7 \mathrm{kDa}$, thus about $18.0 \mathrm{kDa}$ are coming from the oligosaccharides. Neither the glycosylation sites nor the structure of the oligosaccharides are experimentally known for the studied protein. The presence of the oligosaccharides on the protein surface affects the calculated hydrodynamics properties. Watabe et al. have reported both the glycosylation sites and the glycans composition of a similar HER2 protein using MALDI-MS [24]. They have found seven $\mathrm{N}$-glycosylation $\mathrm{NX}(\mathrm{S} / \mathrm{T})$ motifs corresponding to the following asparagine residues: $68,124,187,259,530,571$ and 629. Four of them $(68,259,530$ and 571) were identified as glycosylated in previous X-ray crystallographic studies [11, 25, 26]. Assuming a similar N-glycan composition at each glycosylation site and the extra molecular weight needed to match the experimentally determined value, the following oligosaccharide 
composition is selected as depicted in Scheme 1. The proposed oligosaccharides were derived from those described in the literature [27, 28]. The glycan 1 was added to $\mathrm{ASN}^{68}, \mathrm{ASN}^{124}$, $\mathrm{ASN}^{259}$ and $\mathrm{ASN}^{629}$ whereas the glycan 2 was linked to the remaining $\mathrm{ASN}^{187}, \mathrm{ASN}^{530}$ and $\mathrm{ASN}^{571}$ using the building tools available in the Sybyl-X software [29]. The initial protein model along with the glycan structures are shown in Fig. 1c.

The glycosylated HER2 extracellular domain dimer. The dimeric structure of the HER2 extracellular domain was taken from the Protein Data Bank Server (PDB ID: 3WLW) [14]. The structure of the non-glycosylated monomer defined above was fitted twice on each homodimer chain structure taking into account the protein backbone atoms of the 564 residues common to both fitting structures. The averaged RMSD value on C $\alpha$ atoms was 2.4 and 2.6 $\AA$ for each monomer, respectively. The oligosaccarides were then added on the corresponding ASN residues in a similar way to that described above for the monomer case. The final structure is shown in Fig. 1d.

\subsubsection{Molecular Dynamics Simulations}

AMBER's ff12SB and Glycam-06 force fields were used in all simulations for the protein and oligosaccharides, respectively, as implemented in the Amber 14 suite [30].

The initial models are shown in Table 1 . They were subjected to the following equilibration protocol:

- Structure minimization: The initial structures generated in the previous section were led to a three-stage minimization process: first, a protein-constrained minimization of the oligosaccharide structures, then the protein side-chains with the backbone positions constrained and finally an unconstrained minimization of the full model. At each stage, 100 steepest descent and 400 conjugate gradient cycles were performed. A restrained harmonic potential with a force constant of $50.0 \mathrm{kcal} \cdot \mathrm{mol}^{-1} \cdot \AA^{-2}$ was applied on those constrained positions.

- Solvation: The models were solvated with TIP3P water molecules using the solvateOct method implemented in Amber 14. A truncated octahedral simulation box was built with a minimum distance from solute to any box edge of $12 \AA$ and a closest distance between the glycoprotein and the solvent were as minimum of $1 \AA$. Enough $\mathrm{Na}^{+}$ions are added to neutralize the system charge. Minimization of the water molecules with the constrained glycoprotein positions was carried out to relax bad-contacts between the solvent and the glycoprotein. 
- Equilibration step 1: A 1 ns long NPT-MD simulation was performed on the solvated system at $1 \mathrm{~atm}$. The glycoprotein atomic positions were restrained with a harmonic potential with a force constant of $50.0 \mathrm{kcal} \cdot \mathrm{mol}^{-1} \cdot \AA^{-2}$. The simulation box was evenly heated from $100 \mathrm{~K}$ to $300 \mathrm{~K}$ to thermalize the system.

- Equilibration step 2: A 1 ns long unconstrained NPT-MD simulation on the final structure of the previous phase with a 1 fs time step. The simulation box was evenly heated from $100 \mathrm{~K}$ to $300 \mathrm{~K}$ to thermalize the system.

- Equilibration step 3: A $1 \mathrm{~ns}$ long NPT-MD simulation at T $=300 \mathrm{~K}$ and $\mathrm{P}=1$ atm was run. Then an extra $1 \mathrm{~ns}$ was run saving the structure each $0.1 \mathrm{~ns}$ in separate coordinate files. The eight last structures were used as initial coordinates for each of the replicas.

- Equilibration step 4: A two-step NPT simulation on each of the eight structures saved in the previous step was carried out. The first step consisted of a heating-ramp from $300 \mathrm{~K}$ to $315 \mathrm{~K}$ for a total of $100 \mathrm{ps}$. The second one involved a cooling from $315 \mathrm{~K}$ to $300 \mathrm{~K}$ for another $100 \mathrm{ps}$ period. In these simulations a time step of $2 \mathrm{fs}$ were used. The final density of the equilibration period is around $0.982-0.990 \mathrm{~g} \cdot \mathrm{cm}^{-3}$.

- Production: $50 \mathrm{~ns}$ NVT simulations at $\mathrm{T}=300 \mathrm{~K}$ on each of the final structures saved in the previous step. In these simulations a time step of 2 fs were used.

In all cases, the dynamics simulations were performed using: i) Langevin thermostat with a collision frequency of $2 \mathrm{ps}^{-1}$, ii) Pressure coupling with isotropic position scaling at 1 atm was accomplished using the Berendsen barostat [23] with a pressure relaxation time of 2 ps, iii) a compressibility factor of $44.6 \times 10^{-6} \mathrm{bar}^{-1}$, which is appropriate for water, and iv) SHAKE constraints were used on bond involving hydrogen atoms.

\subsubsection{Hydrodynamic calculations}

The BEST hydrodynamics package (Version 3.9) developed by Prof. S. Aragon was used to calculate the hydrodynamics properties of the previously simulated systems. The BEST package allows for the precise computation of hydrodynamic transport properties of arbitrarily shaped macromolecules subject to the stick boundary condition [31]. This software requires a molecular structure as input. Thus, 25 structures were evenly extracted from each of the $50 \mathrm{~ns} \mathrm{MD}$ production runs described in the previous section. As suggested by Prof. Aragon, $\mathrm{H}$ atoms are removed to get a softer molecular surface for an adequate mathematical treatment. Within this approach the hydrodynamic properties were computed using rigid-body 
(RB) hydrodynamics. The conformational flexibility is taken into account by averaging the hydrodynamic property value for each individual conformation (Fig. S1 in Supplementary Information). This is a classical procedure for flexible molecules within the same framework as the so-called rigid body - Monte Carlo approach (RBMC) with the difference that the generator of macromolecular conformations, instead of Monte Carlo (MC) is replaced by MD of atomic-level models (RBMD) [32-34].

The calculation method involves the following steps: 1) The molecular surface accessible to solvent is defined following Connolly's MSROLL technique [35]. A spherical probe with a radius of $1.6 \AA$ is rolled on the glycoprotein surface defined by the combination of atomic balls with tabulated van der Waals radii inflated by $1.0 \AA$ to take hydration into account. MSROLL outputs a triangulation of the molecular surface which is further refined to eliminate slender triangles and reduce their number (N). 2) This smoothing is accomplished by the program RCOAL, which can compute a varying amount of triangles. Increasing the number of triangles derives on a higher computational cost. A heuristic rule to estimate this maximum is $N=30 \sqrt{M_{w}}$, where $\mathrm{N}$ is the number of triangles and $\mathrm{M}_{\mathrm{w}}$ the molecular weight. For the HER2 monomer case, a minimum of 4,000 triangles and a maximum of 9,000 were considered. Six triangulations were performed between these numbers. For the dimer these values were 5,000 and 12,600, respectively, with four triangulations. 3) The hydrodynamics properties are finally calculated with the BEST program by numerical integration of the Oseen tensor over the triangular surface elements. Among the set of properties that can be obtained from BEST, we were interested in the translational diffusion coefficient, D, and the

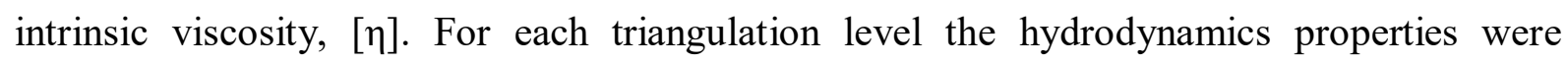
calculated and the final values result from an extrapolation to infinite number of triangles. For this purpose, the property of interest is plotted against $1 / \mathrm{N}$ and the resulting straight line can be fitted so the zero abscissa value is finally extracted (see Fig. S2 in Supplementary Information for the extrapolated values). To compare with the experimental quantities the calculated values are referred to $\mathrm{T}=293 \mathrm{~K}$ with a solvent viscosity of $1.002 \mathrm{mPa} \cdot \mathrm{s}$.

\section{Results and discussion}

\subsection{Experimental hydrodynamic properties}

SEC with tetradetection was used to determine the absolute molecular weight, $\mathrm{M}_{\mathrm{w}}$, and the intrinsic viscosity, $[\eta]$, or alternatively the hydrodynamic radius, $\mathrm{r}_{\mathrm{h}}, \mathrm{SEC}$, in solution of the 
systems under study using Eq (2) (see Figs. 2). For a better visualization of the peaks we have used a log-scale in detector signals in Fig. 2a. This technique allowed us the characterization of individual macromolecules.
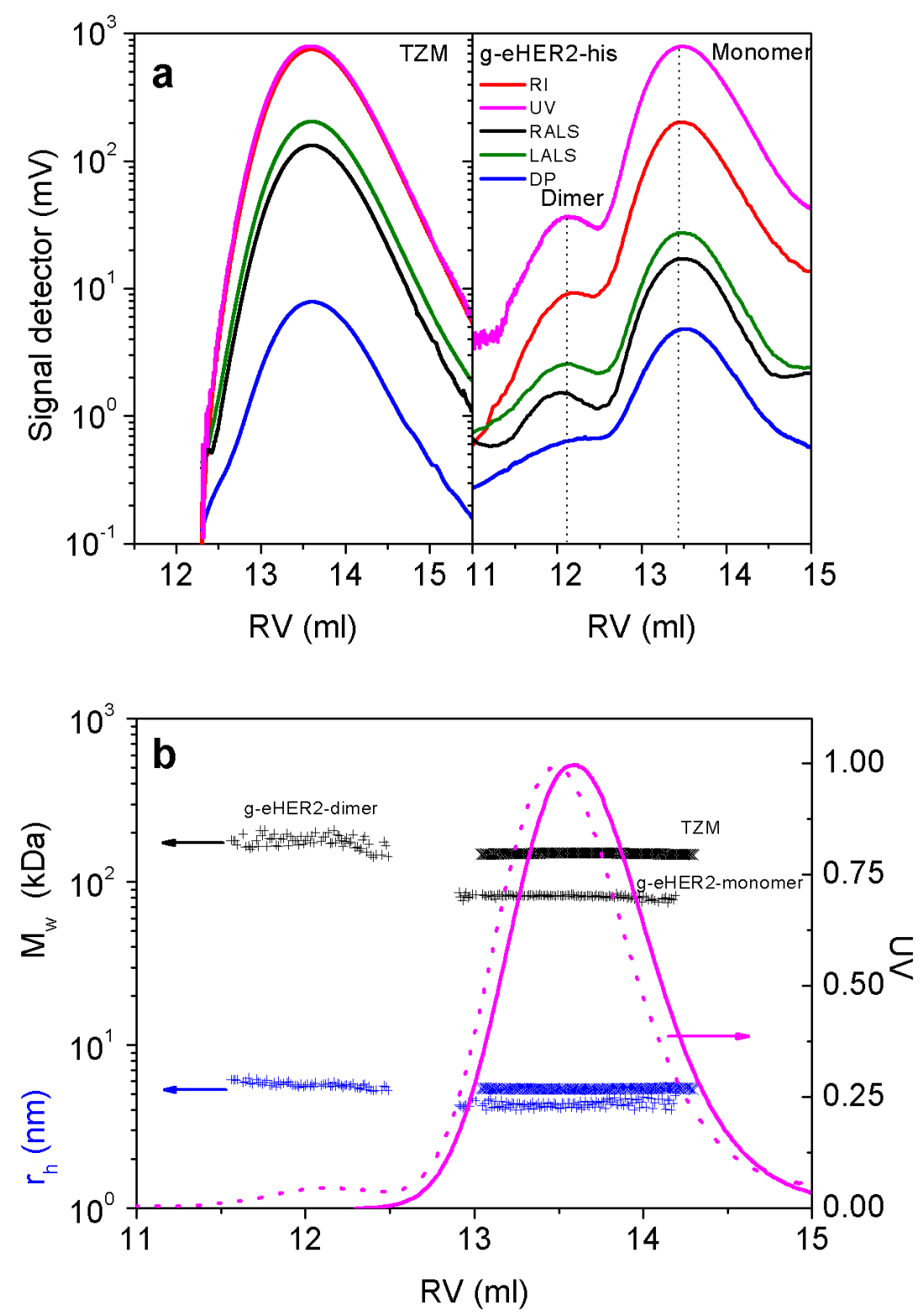

Fig. 2. a) SEC/tetradetection traces of TZM and g-eHER2-his. b) Normalized UV signal (solid line: TZM; dotted line: g-eHER2-his) and measured $\mathrm{M}_{\mathrm{w}}$ (black) and $\mathrm{r}_{\mathrm{h}}$ (blue) of $(\times) \mathrm{TZM}$ and $(+) \mathrm{g}$ eHER2-his mono and dimeric species

A value of $\mathrm{M}_{\mathrm{w}}=149.0 \pm 3.0 \mathrm{kDa}$ for TZM has been measured. The observed TZM molecular weight is in agreement with the typical size of $\mathrm{IgG}$ type antibodies. Concerning geHER2-his and g-eHER2 systems, the major amount of the sample elutes at $13.4 \mathrm{~mL}$, with $\mathrm{M}_{\mathrm{w}}=88.7 \pm 1.8 \mathrm{kDa}$. Primary structure information reveals a predicted monomeric protein 
$\mathrm{M}_{\mathrm{w}}$ of 70.7 and $70.0 \mathrm{kDa}$; it is well-known that EGFR family members are heavily glycosylated [36, 37]. In addition to this, an amount of higher $\mathrm{M}_{\mathrm{w}}$ species $(184.0 \pm 8.7 \mathrm{kDa})$ eluting at $12.1 \mathrm{~mL}$ has been detected. The concentration of this species is around $5 \%$ (for $\mathrm{g}$ eHER2-his) and $15 \%$ (g-eHER2) (Fig. 3). The difference is likely due to the equilibrium between the monomeric and dimeric species and a concentration dependence shift. Higher order oligomer signals are observed in the RALS and LALS traces of the g-eHER2 sample, for elution volumes lower than $11 \mathrm{~mL}$. The $\mathrm{M}_{\mathrm{w}}$ of this very small fraction is not constant, suggesting tiny amounts of oligomeric mixture. It should be noted that in spite of the differences found in the $\mathrm{M}_{\mathrm{w}}$ of TZM and monomeric g-eHER2 species, they elute quite similarly, which might be related to the difference in the compact IgG and open HER2 structures.

The values of $[\eta]$, and consequently of $r_{h, S E C}$, have also been obtained for all the

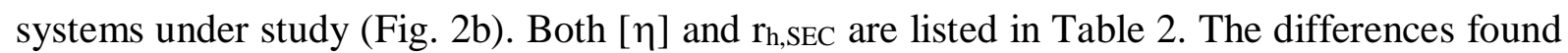
for the values of $M_{w}$ and [ $\eta$ ] between g-eHER2-his and g-eHER2 systems are within the experimental error, but it seems that the absence of the -his tail causes a higher probability of the monomeric species to form the homodimeric ensemble (see Fig. 3).

Table 2. Molecular and hydrodynamic properties of the samples at $T=293 \mathrm{~K}$

\begin{tabular}{|c|c|c|c|c|c|c|}
\hline Sample & $\begin{array}{c}\text { M (kDa) } \\
\text { SEC } \\
\text { (s.d. }<5 \%) \\
\end{array}$ & $\begin{array}{c}\mathbf{r}_{\mathrm{h}}(\mathbf{n m}) \\
\text { SEC }\end{array}$ & $\begin{array}{c}{[\eta]\left(\mathrm{cm}^{3} \cdot \mathrm{g}^{-1}\right)} \\
\text { SEC }\end{array}$ & $\begin{array}{c}\mathbf{r h}_{\mathrm{h}}(\mathbf{n m}) \\
\text { DLS }\end{array}$ & $\begin{array}{c}\mathrm{D} \times 10^{7}\left(\mathrm{~cm}^{2} \cdot \mathrm{s}^{1}\right) \\
\mathrm{DLS}\end{array}$ & $\begin{array}{l}\mathrm{D} \times 10^{7}\left(\mathrm{~cm}^{2} \cdot \mathrm{s}^{-1}\right) \\
\text { Water } 293 \mathrm{~K}^{\mathrm{c}}\end{array}$ \\
\hline$T Z M$ & 149 & $5.4 \pm 0.1$ & $6.5 \pm 0.1$ & $5.2 \pm 0.1$ & $4.01 \pm 0.02$ & $4.09 \pm 0.02$ \\
\hline g-eHER2 & 88.7 & $4.5 \pm 0.1$ & $6.4 \pm 0.2$ & $4.7 \pm 0.1$ & $4.46 \pm 0.02^{\mathrm{b}}$ & $4.56 \pm 0.02$ \\
\hline g-eHER2 dimer & 184.0 & $5.8 \pm 0.2$ & $6.9 \pm 0.5^{a}$ & $6.0 \pm 0.1$ & $3.49 \pm 0.02^{\mathrm{b}}$ & $3.57 \pm 0.02$ \\
\hline
\end{tabular}

It is worth to note the results of the value of [ $\eta]$ obtained for both the monomeric and the homodimeric g-eHER2 species. An increase of nearly twice with respect to that observed for globular proteins in experiments is measured. In general, non-glycosylated globular proteins show values of $[\eta]$ located between 2.5 and $4.5 \mathrm{~cm}^{3} \cdot \mathrm{g}^{-1}$. It is well-known that in these systems, with nearly a spherical shape, $[\eta]$ is lower than for more flexible macromolecules. The theoretical lower limit for $[\eta]$ of a spherical particle obeying stick hydrodynamic boundary conditions is the Einstein value, $[\eta]=2.5 \mathrm{~cm}^{3} \cdot \mathrm{g}^{-1}$. The experimental values obtained for globular proteins are usually slightly above this theoretical limit for an anhydrous sphere, and the difference is due in part to the effect of hydration [38]. Also the shape and flexibility 
of the system play a role in protein hydrodynamics. This is the case of TZM antibody, for which also a considerably higher value of $[\eta]=6.5 \mathrm{~cm}^{3} \cdot \mathrm{g}^{-1}$ than the corresponding to globular proteins is obtained.

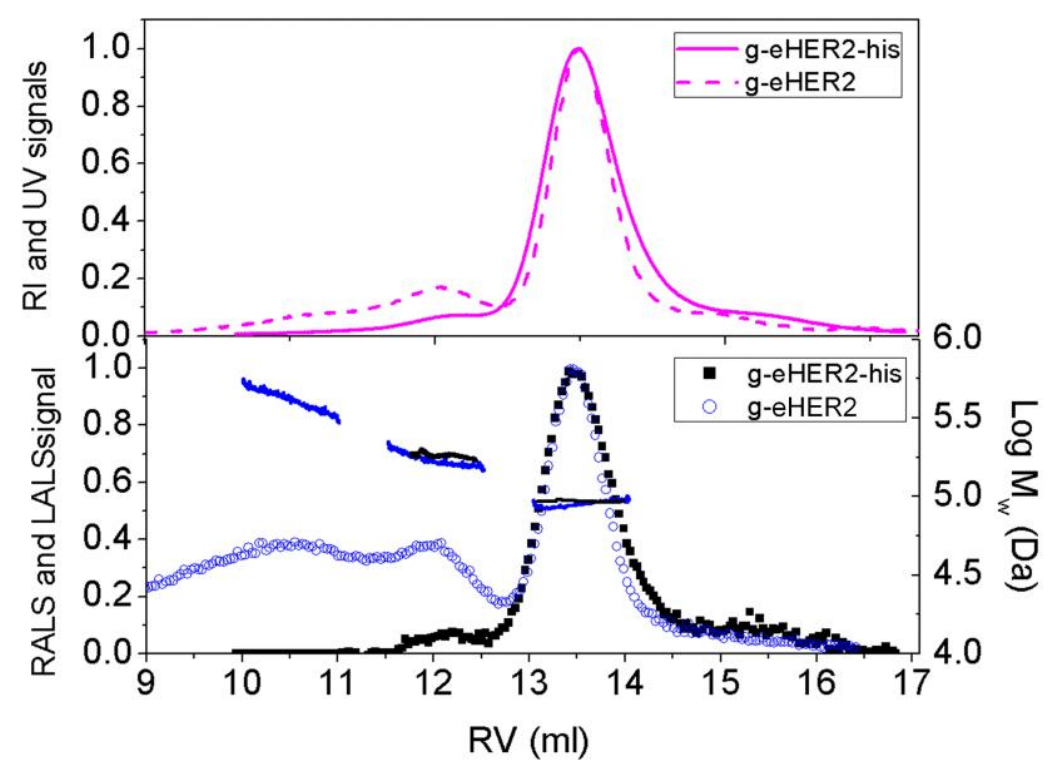

Fig. 3. Normalized IR/UV and RALS/SALS detector signals for g-eHER2-his and g-eHER2 samples. Measured $\mathrm{M}_{\mathrm{w}}$ are also indicated

The case of g-eHER2 monomeric and homodimeric systems is even more complex. On one hand, they contain a considerable amount of glycans. Previous studies have reported the profound effect of heterosaccharide chains on protein conformation [39]. As it occurs in globular simple proteins, $[\eta]$ values of folded glycoproteins are independent of molecular weight. In fact, in glycoproteins as $\alpha-1$ acid glycoprotein $(44.0 \mathrm{kDa}), \alpha-1$ antitrypsin (51.0 $\mathrm{kDa})$ and $\alpha-2$ macroglobulin $(725 \mathrm{kDa})$ one finds reported values of $[\eta]$ of $6.8-6.9 \mathrm{~cm}^{3} \cdot \mathrm{g}^{-1}$ [38-40]. In these specific cases the increase observed in [ $\eta]$ was attributed to the presence of a higher content in sialic acid, affecting the hydrodynamic size, and hence [ $\eta]$, in different ways, i.e. by means of the hydration of the protein or the number of negative charges. Also the asymmetric distribution of the sugars has been suggested as a possible source of the measured difference [39]. The enhanced values of $[\eta]$ found in monomeric and dimeric geHER2 should partially obey to the highly flexible glycans on the surface of the proteins, which increase conspicuously the hydrodynamic size. On the other hand, these systems are characterized by an open molecular conformation (or flexible domain), which should lead to a decrease in molecular density. There is also supporting evidence for the existence of a considerable effect of molecular flexibility due to this open configuration. In fact, our 
previous MD simulations performed on the members of the EGFR family in water, detected large root mean square fluctuations concentrated in domains I, II and IV of the systems, indicating the existence of different movements of the domains and hence a high degree of flexibility [21, 22].

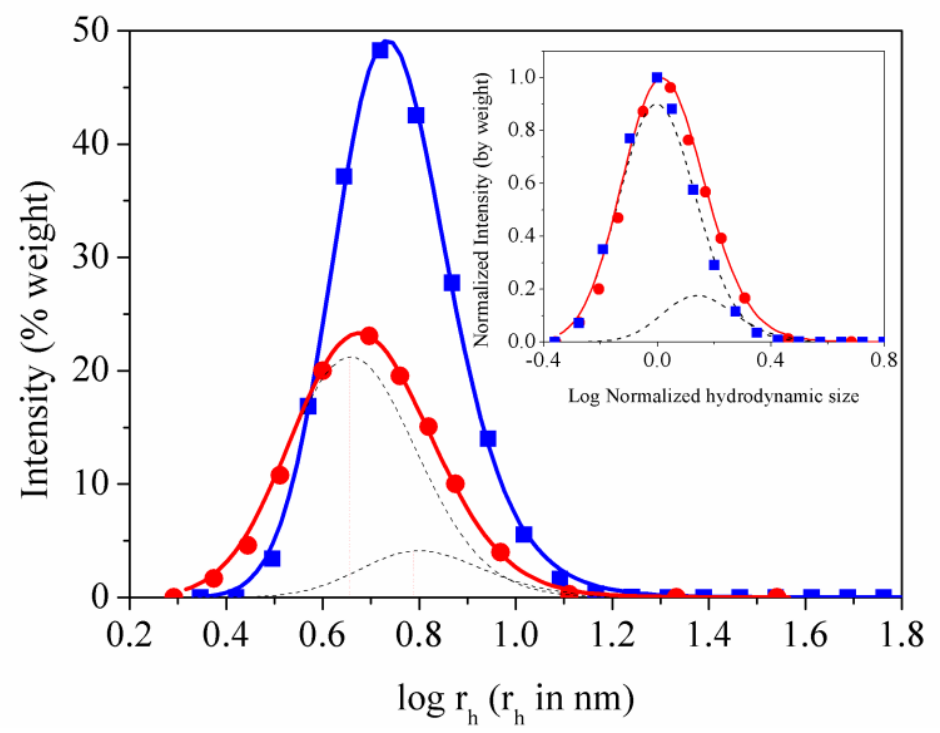

Fig. 4. Mass percent size distributions of the samples studied obtained from DLS experiments: monodisperse TZM (blue squares) and g-eHER2 (red circles). The deconvolution in two monodispersed species of g-eHER2 sample is shown as broken lines. The width of the distributions for monomeric and dimeric species is in agreement to that obtained for monodisperse TZM (insert)

Additionally to $[\eta]$, we have also obtained the values of the translational diffusion coefficient, D, and hence of the hydrodynamic radius, $\mathrm{r}_{\mathrm{h}, \mathrm{DLS}}$ (Eq. 6), by means of DLS experiments. DLS results in Fig. 4, expressed as a function of the hydrodynamic size, assess a clear heterogeneity in g-eHER2 sample when compared to the monodisperse TZM case. This last sample shows the characteristic sharp and intense peak of a monosdisperse species. However, a broader and asymmetric size distribution was obtained for g-eHER2 sample, which comprised two different populations ascribed to the monomeric $\left(\mathrm{D}=4.46 \times 10^{-7} \mathrm{~cm}^{2} \cdot \mathrm{s}^{-}\right.$ ${ }^{1}$, or $\left.\mathrm{r}_{\mathrm{h}, \mathrm{DLS}}=4.7 \mathrm{~nm}\right)$ and dimeric $\left(\mathrm{D}=3.57 \times 10^{-7} \mathrm{~cm}^{2} \cdot \mathrm{s}^{-1}\right.$, or $\left.\mathrm{r}_{\mathrm{h}, \mathrm{DLS}}=6.0 \mathrm{~nm}\right)$ species. This heterogeneity is more clearly envisaged in the normalized curves shown in the inlet to Fig. 4. These results are in agreement with those obtained in the previous SEC experiments, as it can be observed in Table 2. It has been shown in the literature that the equivalent radius obtained in biomacromolecules from different hydrodynamic properties may show differences depending on the shape of the systems. As it was pointed out in section 2.4 , the values obtained for $r_{h}$ from $[\eta]$ and D in the simplest case of an ellipsoid of revolution are nearly the same for a wide range of $p$ values, from flat disks $(p=0.1)$ to long rods $(p=10)$ [19]. In 
the case of highly elongated particles ( $p>>10)$ an increase in the value of $r_{h}$ obtained from [ $\eta$ ] with respect to that obtained from $\mathrm{D}$ is expected (of around $10 \%$ ). The ellipsoidal characterization of the systems studied here has been used to get a description of their approximate shape. We have used the protocol described by Silverman to get the ellipsoidal radii $[41,42]$. Through diagonalization of the second order geometrical tensor we get the moments-of-geometry in an analogous way to the moments-of-inertia. The ellipsoid dimensions are inversely proportional to the root square of the moments-of-geometry. Finally, the aspect ratio between the largest, L, and the smallest, d, ellipsoid dimensions can be obtained calculating the square root of the corresponding moment-of-geometry ratio. Approximate results for the aspect ratios corresponding to the systems studied in this work are: 1.5 for TZM, 2.1 for eHER2, 2.0 for g-eHER2, and 1.4 for g-eHER2 homodimer. The aspect ratio in our systems is then quite low $(\mathrm{p} \leq 2)$ and consequently the calculated $\mathrm{r}_{\mathrm{h}}$ values from $[\eta]$ and D should be nearly the same. The differences found in our systems (see Table 2) are lower than $5 \%$, which is within the uncertainty range of our experimental set-up.

\subsection{Simulations and Hydrodynamic Modeling}

Hydrodynamic modeling offers the possibility of a direct connection between the atomic-level details of MD simulations and transport properties in solution. A number of works concerning the combination of experiments and modeling have been performed in the past for the studies of structural aspects of biomacromolecules [20, 43-56]. To this respect, the methodology proposed by Aragon et al is especially interesting for us. [20, 44, 45, 49]. These authors applied a hydrodynamic modeling approach that permits to compute the properties of the systems from the topological texture of their solvent-accessible surface. This is an ideal method to account for flexible molecules, whose conformations and hence transport properties are expected to fluctuate as they sample their available conformational space. Such an investigation is very valuable in our case, as the results can be straightforward compared to data obtained experimentally. Then, a particular molecular ensemble can be validated against the experiments, making it possible to extract information about the structure and dynamic properties from MD trajectories.

The hydrodynamic properties of the TZM monoclonal antibody were already modeled by Aragon [20]. As a testing case, we followed the simulation protocol described in the previous sections to calculate the hydrodynamic properties of TZM and compare with the corresponding experimental data obtained at our lab. The average translational diffusion 
coefficient and intrinsic viscosity are compared with the experimentally derived quantities in Table 3.

Table 3. Comparison of experimental and calculated hydrodynamic results for the structures studied, including the TZM as model case, at $T=293 \mathrm{~K}$

\begin{tabular}{|c|c|c|c|c|}
\hline Sample & $\begin{array}{c}{[\eta]\left(\mathrm{cm}^{3} \cdot \mathrm{g}^{-1}\right)} \\
\text { SEC }\end{array}$ & $\begin{array}{c}{[\eta]\left(\mathrm{cm}^{3} \cdot \mathrm{g}^{-1}\right)} \\
\text { SIM }\end{array}$ & $\begin{array}{c}\mathrm{D} \times 10^{7}\left(\mathrm{~cm}^{2} \cdot \mathrm{s}^{-1}\right)^{\mathrm{a}} \\
\mathrm{DLS}\end{array}$ & $\begin{array}{c}\mathrm{D} \times 10^{7}\left(\mathrm{~cm}^{2} \cdot \mathrm{s}^{-1}\right)^{\mathrm{a}} \\
\mathrm{SIM}\end{array}$ \\
\hline$T Z M$ & $6.5 \pm 0.1$ & $6.6 \pm 0.2$ & $4.09 \pm 0.02(5.2 \mathrm{~nm})$ & $3.95 \pm 0.03(5.3 \mathrm{~nm})$ \\
\hline eHER2 & n.d. & $5.7 \pm 0.2$ & n.d. & $5.32 \pm 0.04(4.0 \mathrm{~nm})$ \\
\hline g-eHER2 & $6.4 \pm 0.2$ & $6.5 \pm 0.2$ & $4.56 \pm 0.02(4.7 \mathrm{~nm})$ & $4.77 \pm 0.04(4.5 \mathrm{~nm})$ \\
\hline g-eHER2 dimer & $6.9 \pm 0.5$ & $6.9 \pm 0.2$ & $3.57 \pm 0.02(6.0 \mathrm{~nm})$ & $3.71 \pm 0.02(5.8 \mathrm{~nm})$ \\
\hline
\end{tabular}

As can be observed the simulated values are in a very good agreement with both the experimental $(\varepsilon=1.5 \%$ and $3.4 \%$ for the intrinsic viscosity and the translational diffusion coefficient, respectively) results and the simulated values given by Aragon et al. $\left(3.95 \times 10^{-7}\right.$ vs $4.08 \times 10^{-7} \mathrm{~cm}^{2} \cdot \mathrm{s}^{-1}$ for translational diffusion coefficient and $6.6 v s 6.2 \mathrm{~cm}^{3} \cdot \mathrm{g}^{-1}$ for intrinsic viscosity) [20].

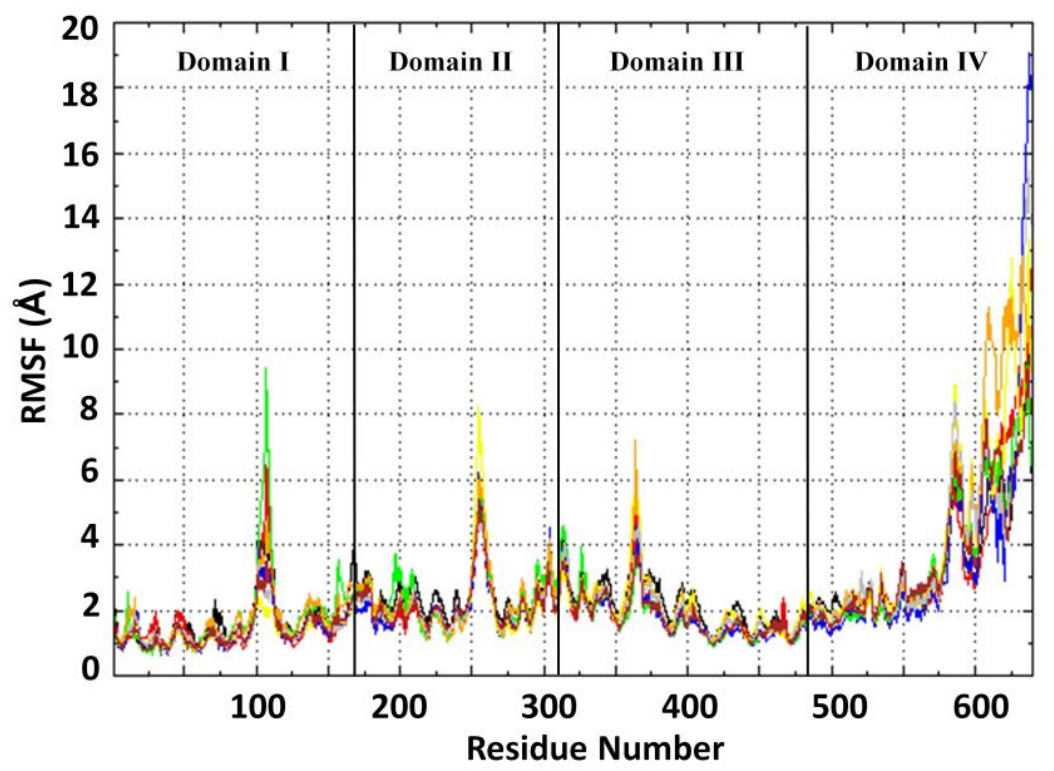

Fig. 5. Root mean squared fluctuation per residue (RMSF) of the g-eHER2 during the NVT simulations (each color represent a different replica)

The simulated structure of the non-glycosylated eHER2 monomer is similar to that studied in our previous publication, where the OPLS-AA instead of Amber Force Field was used [21, 22]. The most notable characteristic, also observed in the OPLS-AA model, is the hinge in the loop region between domains III and IV which likely results from the proximity of both domains. The additional 23 residues and the poly-HIS chain at the protein N-terminus 
of the present model respect to the model in [21] does not significantly modify the overall structure of the monomer. In Table 4 the per-domain root-mean-square fluctuation (RMSF) calculated over the $\mathrm{C} \alpha$ atoms is presented.

Table 4. RMSF values $(\AA)$ for the different HER2 extracellular domains

\begin{tabular}{ccccccc}
\hline Domains & I & II & III & IV & I-III & All \\
\hline $\boldsymbol{e H E R 2}$ & $2.1 \pm 0.4$ & $2.4 \pm 0.4$ & $1.9 \pm 0.3$ & $4.3 \pm 0.8$ & $2.6 \pm 0.3$ & $4.3 \pm 0.7$ \\
g-eHER2 & $1.8 \pm 0.3$ & $2.2 \pm 0.3$ & $2.0 \pm 0.3$ & $3.7 \pm 0.7$ & $2.4 \pm 0.3$ & $3.9 \pm 0.7$ \\
\hline
\end{tabular}

The average coordinate fluctuation for each residue corresponding to different replicas is plotted in Fig. 5. As can be observed, the most flexible domain of the protein is the domain IV, in agreement with previously reported results [21, 22]. The averaged translational diffusion coefficient and intrinsic viscosity can be compared with the experimentally derived in Table 3. The simulated values indicate faster diffusion $\left(5.32 \times 10^{-7} v s 4.56 \times 10^{-7} \mathrm{~cm}^{2} \cdot \mathrm{s}^{-1}\right)$ and lower viscosity $\left(5.7 v s 6.4 \mathrm{~cm}^{3} \mathrm{~g}^{-1}\right)$ compared to the experimental results. It is worth to mention that the flexibility of domain IV is likely due to its intrinsically disordered structure $[11,14,57]$. Domain IV of HER2 is of great interest, as it has been shown that TZM binds to this region, identifying this site as a target for anticancer therapies [58]. It should be also noted that although the structures studied here display an interesting conformational variability (see Fig. S1 in Supplementary Information), the change in calculated hydrodynamic properties among them is moderate. In fact the conformational averages determined from the different MD trajectories have standard deviations of less than $3 \%$ (see Supplemental Information).

The next step is to add the glycan to the protein surface, as explained in the Materials and Methods (Computational simulations section), in order to evaluate if the presence of the glycan on the protein surface has any influence on the hydrodynamics properties. Firstly, the addition of the oligosaccharides to the seven glycosylation sites has a negligible influence in the overall monomer structure (see Fig. 1c). The corresponding RMSD values categorized by protein domains and averaged over all the molecular dynamics trajectories are presented in Table 4. The largest value corresponds again to domain IV, although the absolute quantities are slightly lower than for the non-glycosylated protein. More important effect is the influence of glycans on the hydrodynamics properties, which are reported in Table 3. As can be observed, the translational diffusion coefficient $\left(4.77 \times 10^{-7}\right.$ vs $\left.4.56 \times 10^{-7} \mathrm{~cm}^{2} \cdot \mathrm{s}^{-1}, \varepsilon=4.6 \%\right)$ as well as the intrinsic viscosity $\left(6.5 v s 6.4 \mathrm{~cm}^{3} \cdot \mathrm{g}^{-1}, \varepsilon=1.6 \%\right)$ compare more satisfactorily 
well with the experimental values. Therefore, the addition of glycan chains to the HER2 structure seems to be essential to explain the experimentally observed transport properties.

It is well-known that the dimerization of the extracellular domains is a key step in the signaling path of the EGFRs family. Recently, $\mathrm{Hu}$ et al. have crystallized an HER2 ECD homodimer, which is distinctive compared with other EGFR dimer models [14]. The structure of the eHER2 homodimer consists of a "back-to-head" interaction of the two protomers. Thus, we have built a g-eHER2 homodimer model based on this crystallographic structure, using the glycosylation pattern already described. The structure of the crystallographic homodimer is largely preserved along the molecular dynamics simulations. As an example, the contact map of all trajectories is shown in Fig. 6. This map has been calculated using the MDcons tool [59]. The plot shows the residue-residue contacts between domain II of one protomer (protomer 2 in Fig. 6) and domain III of the other (protomer 1 in Fig. 6). Each point means a residue contact with a persistent distance (larger than $90 \%$ in the MD trajectory) below $5 \AA$ between the corresponding centers of mass. It can be concluded that the contacts between the two monomers are mostly unperturbed by the oligosaccharides because they remain outside the contacting subdomains. The superposition between the averaged-MD simulation and the crystallographic structures shows that the structures are very similar (RMSF of the backbone atoms around $4 \AA$ ).

a)

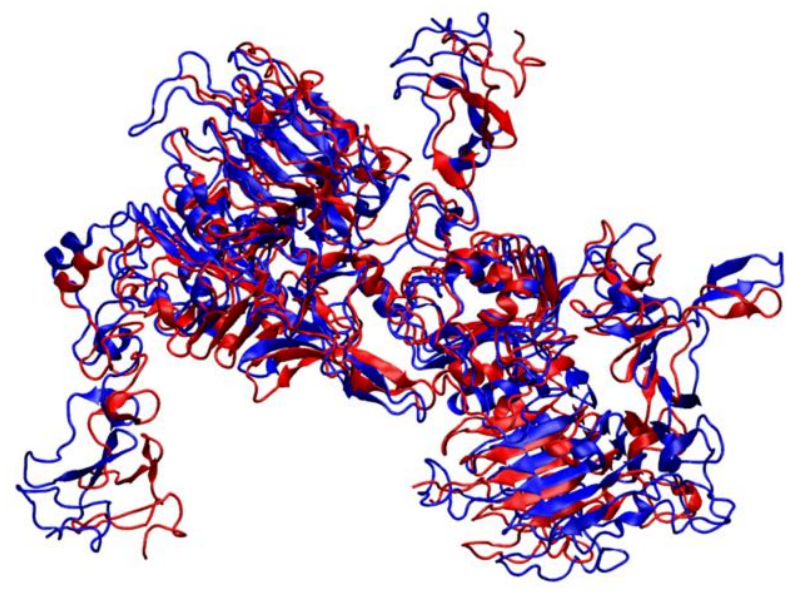

b)

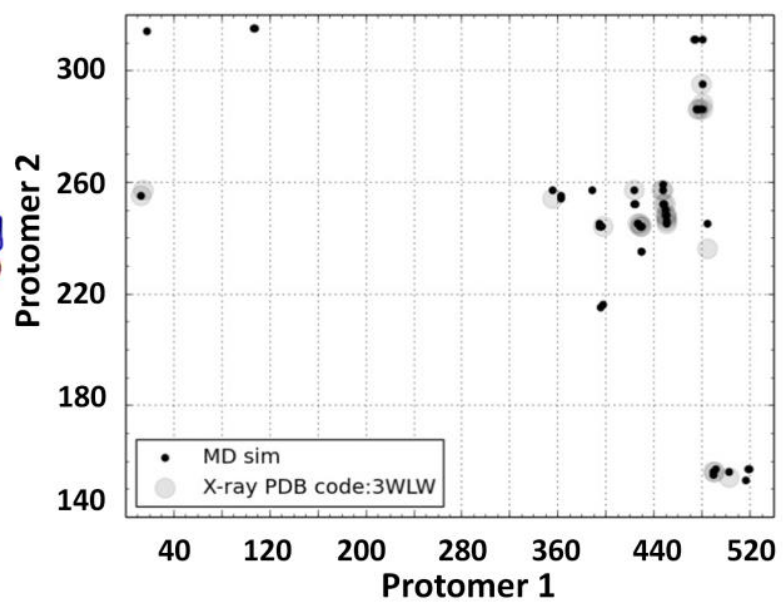

Fig. 6. a) Superimposed structures of the crystallographic eHER2 dimer (red, PDB ID: 3WLW) and a selected last structure of the simulations (blue). b) Intermolecular contact map of the eHER2 dimer Xray structure (gray points) and intermolecular contact map of MD snapshots from the eight replicas (black points). The contact maps were calculated using the MDcons software [59]

The calculated hydrodynamic properties are tabulated in Table 3 along with the experimental data. These results show a nice agreement between the experimental 
determinations and the computational hydrodynamic analysis of the collection of MD trajectories samples for TZM and g-eHER2-his monomer and homodimer. Therefore, the crystallographic homodimer structure for the eHER2 complemented by the set of oligosaccharide glycosylations is able to explain the transport properties observed for this complex. The percentage difference between the calculated and experimental values determined from the experimental average are less than $5 \%$. These results support that this approach is appropriate, not only in the cases of flexible antibodies and globular proteins [20, 44, 45, 49], but also for heavily glycosylated flexible proteins such as monomeric and homodimeric g-eHER2 structures studied here.
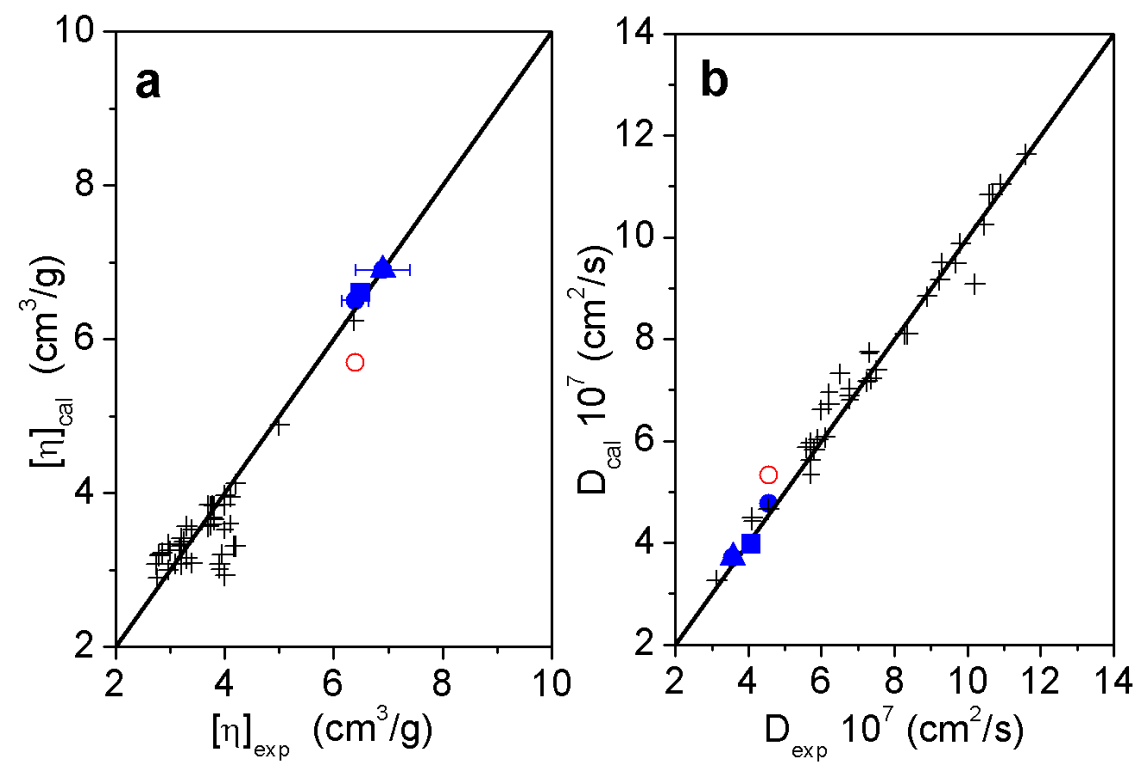

Fig.7. Comparison between calculated and experimental values of intrinsic viscosity and translational

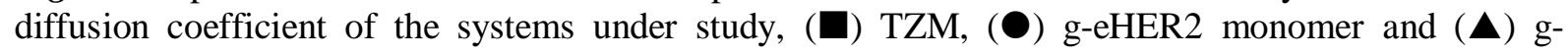
eHER2 dimer, $(\bigcirc)$ eHER2-his (non-glycosylated) and $(+)$ mono and multimeric globular proteins taken from the literature $[20,44,45,49]$

In Fig. 7 we show a comparison of the calculated and the experimental values of [ $\eta]$ and D obtained for our systems and for other taken from the literature (mostly globular monomeric and multimeric proteins). As it can be observed, the values of [ $\eta$ ] (Fig. 7a) in our systems (solid symbols) fit perfectly to the experimental values, and they are nearly twice as those corresponding to globular proteins. The values of D obtained are the lowest ones within the range explored in previous works, and significantly lower than those expected for globular systems with the same $M_{w}$ (Fig. $7 b$ ). We have included in the graphs the results obtained for the simulation in non-glycosylated eHER2 system (open symbol). As judged by the deviation found, it can be deduced that most of the changes found in the hydrodynamic properties for $\mathrm{g}$ eHER2-his monomer and homodimer with respect to those values found in globular proteins, 
around $70 \%$ of the change being due to its specific shape, conformational features and flexibility, whereas $30 \%$ of the change the glycans.

\section{Conclusions}

In this work we describe a combination of experiments and simulations of molecular dynamics and transport properties of the HER2 extracellular domain and its homodimer in aqueous solution. Our results indicate good agreement between experimental results and simulation-derived parameter, supporting a high degree of molecular flexibility in both monomeric and dimeric species, which ultimately leads to quite higher values of the intrinsic viscosity and lower values of diffusion coefficient than those corresponding to simple globular proteins of similar molecular mass. The simulations indicate that the homodimer in solution is in part firmly assembled, but it retains in other parts the interesting and specific dynamic features of the monomeric species. This high flexibility stem from the open conformation of this specific receptor and from the large root mean square fluctuations of the different domains, especially the domain IV due to its hinge movement, previously reported by us. Finally, we demonstrated that for obtaining the correct values of hydrodynamic constants from computer simulations one must consider the glycosylation of the systems. This work not only provides new insights about the unique structural properties of this important protein in solution, but also elucidates the usefulness of experimental approaches.

\section{Acknowledgments}

This research work was supported by Spanish Ministry of Economy and Competitiveness (MINECO, Spain) - Project MAT2012-36341-FEDER and by the CSIC (Spain) - Project PIE201360E097. J. Ramos acknowledges financial support through the Ramón y Cajal program (MINECO, Spain) - Contract RYC-2011-09585. We acknowledge Prof. Sergio R. Aragón (San Francisco State University, USA) for kindly providing us the BEST software to compute the transport properties of biomacromolecules. We also acknowledge Maurizio Scaltriti (Memorial Sloan Kettering Cancer Center, New York, USA) for helpful discussions.

\section{References}

[1] M.J. Wieduwilt, M.M. Moasser, The epidermal growth factor receptor family: Biology driving targeted therapeutics, Cel. Mol. Life Sci., 65 (2008) 1566-1584. 
[2] A.W. Burgess, H.-S. Cho, C. Eigenbrot, K.M. Ferguson, T.P.J. Garrett, D.J. Leahy, M.A. Lemmon, M.X. Sliwkowski, C.W. Ward, S. Yokoyama, An open-and-shut case? Recent insights into the activation of egf/erbb receptors, Mol. Cell, 12 (2003) 541-552.

[3] M.A. Lemmon, Ligand-induced erbb receptor dimerization, Exp. Cell Res., 315 (2009) 638-648.

[4] R.-H. Tao, I.N. Maruyama, All egf(erbb) receptors have preformed homo- and heterodimeric structures in living cells, J. Cell Sci., 121 (2008) 3207-3217.

[5] P.J. Brennan, T. Kumogai, A. Berezov, R. Murali, M.I. Greene, Her2/neu: Mechanisms of dimerization/oligomerization, Oncogene, 19 (2000) 6093-6101.

[6] L.N. Klapper, S. Glathe, N. Vaisman, N.E. Hynes, G.C. Andrews, M. Sela, Y. Yarden, The erbb-2/her2 oncoprotein of human carcinomas may function solely as a shared coreceptor for multiple stromaderived growth factors, Proc. Natl. Acad. Sci. USA, 96 (1999) 4995-5000.

[7] D. Karunagaran, E. Tzahar, R.R. Beerli, X. Chen, D. Graus-Porta, B.J. Ratzkin, R. Seger, N.E. Hynes, Y. Yarden, Erbb-2 is a common auxiliary subunit of ndf and egf receptors: Implications for breast cancer, The Embo J., 15 (1996) 254-264.

[8] A.W. Burgess, Egfr family: Structure physiology signalling and therapeutic targets, Growth Factors, 26 (2008) 263-274.

[9] A. Badache, N.E. Hynes, A new therapeutic antibody masks erbb2 to its partners, Cancer Cell, 5 (2004) 299-301.

[10] A. Arkhipov, Y. Shan, E.T. Kim, R.O. Dror, D.E. Shaw, Her2 activation mechanism reflects evolutionary preservation of asymmetric ectodomain dimers in the human egfr family, elife, 2 (2013) e00708.

[11] M.C. Franklin, K.D. Carey, F.F. Vajdos, D.J. Leahy, A.M. de Vos, M.X. Sliwkowski, Insights into erbb signaling from the structure of the erbb2-pertuzumab complex, Cancer Cell, 5 (2004) 317-328.

[12] T.P.J. Garrett, N.M. McKern, M. Lou, T.C. Elleman, T.E. Adams, G.O. Lovrecz, M. Kofler, R.N. Jorissen, E.C. Nice, A.W. Burgess, C.W. Ward, The crystal structure of a truncated erbb2 ectodomain reveals an active conformation, poised to interact with other erbb receptors, Mol. Cell, 11 (2003) 495-505.

[13] E. Kovacs, J.A. Zorn, Y. Huang, T. Barros, J. Kuriyan, A structural perspective on the regulation of the egf receptor, Ann. Rev. Biochem., 84 (2015) 739-764.

[14] S. Hu, Y. Sun, Y. Meng, X. Wang, W. Yang, W. Fu, H. Guo, W. Qian, S. Hou, B. Li, Z. Rao, Z. Lou, Y. Guo, Molecular architecture of the erbb2 extracellular domain homodimer, Oncotarget, 6 (2015) 1695-1706.

[15] B.J. Berne, R. Pecora, Dynamic light scattering with application to chemistry, biology and physics, Dover Publications, Inc., Mineola (NY), 2000.

[16] S.W. Provencher, A constrained regularization method for inverting data represented by linear algebraic or integral equations, Comp. Phys. Comm., 27 (1982) 213-227.

[17] G. Mie, Beiträge zur optik trüber medien, speziell kolloidaler metallösungen, Annalen der Physik, 330 (1908) 377-445.

[18] J. Stetefeld, S.A. McKenna, T.R. Patel, Dynamic light scattering: A practical guide and applications in biomedical sciences, Biophys. Rev., 8 (2016) 409-427.

[19] A. Ortega, J. García de la Torre, Equivalent radii and ratios of radii from solution properties as indicators of macromolecular conformation, shape, and flexibility, Biomacromolecules, 8 (2007) 2464-2475.

[20] J.P. Brandt, T.W. Patapoff, S.R. Aragon, Construction, md simulation, and hydrodynamic validation of an all-atom model of a monoclonal igg antibody, Biophys. J., 99 (2010) 905-913.

[21] J.F. Franco-Gonzalez, V.L. Cruz, J. Ramos, J. Martínez-Salazar, Conformational flexibility of the erbb2 ectodomain and trastuzumab antibody complex as revealed by molecular dynamics and principal component analysis, J. Mol. Mod., 19 (2013) 1227-1236.

[22] J.F. Franco-Gonzalez, J. Ramos, V.L. Cruz, J. Martínez-Salazar, Simulation of homology models for the extracellular domains (ecd) of erbb3, erbb4 and the erbb2-erbb3 complex in their active conformations, J. Mol. Mod., 19 (2013) 931-941. 
[23] H.J.C. Berendsen, J.P.M. Postma, W.F. Vangunsteren, A. Dinola, J.R. Haak, Molecular-dynamics with coupling to an external bath, Journal of Chemical Physics, 81 (1984) 3684-3690.

[24] M. Watanabe, K. Terasawa, K. Kaneshiro, H. Uchimura, R. Yamamoto, Y. Fukuyama, K. Shimizu, T.-A. Sato, K. Tanaka, Improvement of mass spectrometry analysis of glycoproteins by maldi-ms using 3-aminoquinoline/ $\alpha$-cyano-4-hydroxycinnamic acid, Analytical and Bioanalytical Chemistry, 405 (2013) 4289-4293.

[25] J. Bostrom, S.-F. Yu, D. Kan, B.A. Appleton, C.V. Lee, K. Billeci, W. Man, F. Peale, S. Ross, C. Wiesmann, G. Fuh, Variants of the antibody herceptin that interact with her2 and vegf at the antigen binding site, Science, 323 (2009) 1610-1614.

[26] C. Eigenbrot, M. Ultsch, A. Dubnovitsky, L. Abrahmsen, T. Hard, Structural basis for high-affinity her2 receptor binding by an engineered protein, Proceedings of the National Academy of Sciences of the United States of America, 107 (2010) 15039-15044.

[27] K.D. Smith, M.J. Davies, D. Bailey, D.V. Renouf, E.F. Hounsell, Analysis of the glycosylation patterns of the extracellular domain of the epidermal growth factor receptor expressed in chinese hamster ovary fibroblasts, Growth Factors, 13 (1996) 121-132.

[28] Y.J. Zhen, R.M. Caprioli, J.V. Staros, Characterization of glycosylation sites of the epidermal growth factor receptor, Biochemistry, 42 (2003) 5478-5492.

[29] TRIPOS Associates Inc., Sybyl-x molecular modeling software packages, in, St. Louis, MO, USA, 2012.

[30] V.B. D.A. Case, J.T. Berryman, R.M. Betz, Q. Cai, D.S. Cerutti, T.E. Cheatham, III, T.A. Darden, R.E. Duke, H. Gohlke, A.W. Goetz, S. Gusarov, N. Homeyer, P. Janowski, J. Kaus, I. Kolossváry, A. Kovalenko, T.S. Lee, S. LeGrand, T. Luchko, R. Luo, B. Madej, K.M. Merz, F. Paesani, D.R. Roe, A. Roitberg, C. Sagui,R. Salomon-Ferrer, G. Seabra, C.L. Simmerling, W. Smith, J. Swails, R.C. Walker, J. Wang, R.M. Wolf, X. Wu and P.A. Kollman, Amber 14, in, University of California, San Francisco, 2014. [31] S. Aragon, A precise boundary element method for macromolecular transport properties, Journal of Computational Chemistry, 25 (2004) 1191-1205.

[32] P.J. Hagerman, B.H. Zimm, Monte carlo approach to the analysis of the rotational diffusion of wormlike chains, Biopolymers, 20 (1981) 1481-1502.

[33] A. Iniesta, F.G. Díaz, J. García de la Torre, Transport properties of rigid bent-rod macromolecules and of semiflexible broken rods in the rigid-body treatment. Analysis of the flexibility of myosin rod, Biophys. J., 54 (1988) 269-275.

[34] J.G. de la Torre, Á. Ortega, D. Amorós, R.R. Schmidt, J.G.H. Cifre, Methods and tools for the prediction of hydrodynamic coefficients and other solution properties of flexible macromolecules in solution. A tutorial minireview, Macromolecular Bioscience, 10 (2010) 721-730.

[35] M.L. Connolly, The molecular-surface package, Journal of Molecular Graphics, 11 (1993) 139143.

[36] A. Ullrich, L. Coussens, J.S. Hayflick, T.J. Dull, A. Gray, A.W. Tam, J. Lee, Y. Yarden, T.A. Libermann, J. Schlessinger, J. Downward, E.L.V. Mayes, N. Whittle, M.D. Waterfield, P.H. Seeburg, Human epidermal growth factor receptor cdna sequence and aberrant expression of the amplified gene in a431 epidermoid carcinoma cells, Nature, 309 (1984) 418-425.

[37] M. Watanabe, K. Terasawa, K. Kaneshiro, H. Uchimura, R. Yamamoto, Y. Fukuyama, K. Shimizu, T.A. Sato, K. Tanaka, Improvement of mass spectrometry analysis of glycoproteins by maldi-ms using 3-aminoquinoline/alpha-cyano-4-hydroxycinnamic acid, Analytical and Bioanalytical Chemistry, 405 (2013) 4289-4293.

[38] S.E. Harding, The intrinsic viscosity of biological macromolecules. Progress in measurement, interpretation and application to structure in dilute solution, Prog. Biophys. Mol. Biol., 68 (1997) 207262.

[39] F. Ahmad, P. McPhie, The intrinsic viscosity of glycoproteins, Int. J. Biochem., 11 (1980) 91-96.

[40] J.K. Armstrong, R.B. Wenby, H.J. Meiselman, T.C. Fisher, The hydrodynamic radii of macromolecules and their effect on red blood cell aggregation, Biophys. J., 87 (2004) 4259-4270.

[41] B.D. Silverman, Asymmetry in the burial of hydrophobic residues along the histone chains of eukarya, archaea and a transcription factor, BMC Structural Biology, 5 (2005) 20-20. 
[42] B.D. Silverman, Using molecular principal axes for structural comparison: Determining the tertiary changes of a fab antibody domain induced by antigenic binding, BMC Structural Biology, 7 (2007) 77-77.

[43] D. Amorós, A. Ortega, S.E. Harding, J. García de la Torre, Multi-scale calculation and global-fit analysis of hydrodynamic properties of biological macromolecules: Determination of the overall conformation of antibody igg molecules, Eur. Biophys. J., 39 (2010) 361-370.

[44] S.R. Aragon, Recent advances in macromolecular hydrodynamic modelong, Methods, 54 (2011) 101-114.

[45] S.R. Aragon, Accurate hydrodynamic modeling with the boundary element method, in: S. Uchiyama, F. Arisaka, W.F. Stafford, T. Laue (Eds.) Analytical ultracentrifugation: Instrumentation, software, and applications, Springer Japan, Tokyo, 2016, pp. 219-247.

[46] M.K. Boehm, J.M. Woof, M.A. Kerr, S.J. Perkins, The fab and fc fragments of iga1 exhibit a different arrangement from that in igg: A study by $\mathrm{x}$-ray and neutron solution scattering and homology modelling, J. Mol. Biol., 286 (1999) 1421-1447.

[47] P.B. Furtado, P.W. Whitty, A. Robertson, J.T. Eaton, A. Almogren, M.A. Kerr, J.M. Woof, S.J. Perkins, Solution structure determination of monomeric human iga2 by $\mathrm{x}$-ray and neutron scattering, analytical ultracentrifugation and constrained modelling: A comparison with monomeric human iga1, J. Mol. Biol., 338 (2004) 921-941.

[48] J. García de la Torre, S.E. Harding, Hydrodynamic modelling of protein conformation in solution: Ellips and hydro, Biophys. Rev., 5 (2013) 195-206.

[49] D.K. Hahn, S.R. Aragon, Intrinsic viscosity of proteins and platonic solids by boundary element methods, J. Chem. Theor. Comp., 2 (2006) 1416-1428.

[50] E. Longman, K. Kreusel, S.B. Tendler, I. Fiebrig, K. King, J. Adair, P. O'Shea, A. Ortega, J. Garcia de la Torre, S.E. Harding, Estimating domain orientation of two human antibody igg4 chimeras by crystallohydrodynamics, Eur. Biophys. J., 32 (2003) 503-510.

[51] Y. Lu, S.E. Harding, T.E. Michaelsen, E. Longman, K.G. Davis, Á. Ortega, J.G. Grossmann, I. Sandlie, J. García de la Torre, Solution conformation of wild-type and mutant igg3 and igg4 immunoglobulins using crystallohydrodynamics: Possible implications for complement activation, Biophys. J., 93 (2007) 3733-3744.

[52] Y. Lu, E. Longman, K.G. Davis, Á. Ortega, J.G. Grossmann, T.E. Michaelsen, J.G. de la Torre, S.E. Harding, Crystallohydrodynamics of protein assemblies: Combining sedimentation, viscometry, and $\mathrm{x}$ ray scattering, Biophys. J., 91 (2006) 1688-1697.

[53] Z. Sun, A. Almogren, P.B. Furtado, B. Chowdhury, M.A. Kerr, S.J. Perkins, Semi-extended solution structure of human myeloma immunoglobulin d determined by constrained $\mathrm{x}$-ray scattering, J. Mol. Biol., 353 (2005) 155-173.

[54] E. Brookes, B. Demeler, C. Rosano, M. Rocco, The implementation of somo (solution modeller) in the ultrascan analytical ultracentrifugation data analysis suite: Enhanced capabilities allow the reliable hydrodynamic modeling of virtually any kind of biomacromolecule, Eur. Biophys. J., 39 (2010) 423-435.

[55] E. Brookes, B. Demeler, M. Rocco, Developments in the us-somo bead modeling suite: New features in the direct residue-to-bead method, improved grid routines, and influence of accessible surface area screening, Macromolecular Bioscience, 10 (2010) 746-753.

[56] M. Rocco, E. Brookes, Dynamical aspects of biomacromolecular multi-resolution modelling using the ultrascan solution modeler (us-somo) suite, in: J.A.K. Howard, H.A. Sparkes, P.R. Raithby, A.V. Churakov (Eds.) The future of dynamic structural science, Springer Netherlands, Dordrecht, 2014, pp. 189-199.

[57] E. Lobner, A.-S. Humm, K. Göritzer, G. Mlynek, M.G. Puchinger, C. Hasenhindl, F. Rüker, M.W. TraxImayr, K. Djinović-Carugo, C. Obinger, Fcab-her2 interaction: A ménage à trois. Lessons from Xray and solution studies, Structure.

[58] H.-S. Cho, K. Mason, K.X. Ramyar, A.M. Stanley, S.B. Gabelli, D.W. Denney, D.J. Leahy, Structure of the extracellular region of her2 alone and in complex with the herceptin fab, Nature, 421 (2003) 756-760. 
[59] S. Abdel-Azeim, E. Chermak, A. Vangone, R. Oliva, L. Cavallo, Mdcons: Intermolecular contact maps as a tool to analyze the interface of protein complexes from molecular dynamics trajectories, BMC Bioinformatics, 15 (2014) S1-S1. 